\title{
Temperature accuracy and temperature gradients in solution-state NMR spectrometers
}

\author{
Nikolaus M. Loening ${ }^{1}$ and James Keeler* \\ Department of Chemistry, University of Cambridge, Lensfield Road, Cambridge CB2 1EW, UK
}

Received 1 May 2002; revised 1 July 2002

\begin{abstract}
In solution-state NMR spectrometers, there is a systematic deviation between the temperature of the sample and the temperature reported by the spectrometer. In addition, temperature gradients are often present in the sample. The size of both the temperature deviations and the temperature gradients depends on several factors, including the temperature, the flow rate of the heating/cooling gas, and the amount of radiofrequency heating.
\end{abstract}

(c) 2002 Elsevier Science (USA). All rights reserved.

Keywords: NMR; Temperature; Temperature gradient

\section{Introduction}

In this paper, we show that there is a significant difference between the actual temperature of the sample and the temperature reported by the spectrometer. This difference is a function of the gas flow rate, the temperature, and the amount of radiofrequency irradiation of the sample; under some circumstances the difference can be several degrees. By using an imaging method, we have also investigated the range of temperatures that are present in the sample. Not surprisingly, the range of temperatures depends on the same parameters as the temperature deviation.

Since physical parameters, such as rate constants and diffusion coefficients, are generally temperature dependent, it is essential to know the temperature in order to report the value of these parameters in a meaningful way. The deviation of the sample temperature from the temperature reported by the spectrometer (which we term the system temperature) causes a problem in this regard, and sets a limit on the confidence that we can have in our values.

\footnotetext{
${ }^{*}$ Corresponding author. Fax: +44-1223-336913.

E-mail address: jhk10@cam.ac.uk (J. Keeler).

${ }^{1}$ Current address: MIT/Harvard Center for Magnetic Resonance, 150 Albany St., Cambridge, MA 02139, USA.
}

The presence of temperature gradients also has an effect on the measured value of a physical parameter as different positions in the sample correspond to different values. However, the value of most physical parameters is linear with temperature over the relatively small range of temperatures typically found in the sample. It therefore follows that the measured value of the parameter is characteristic of the average temperature of the sample; the presence of a temperature gradient will not appreciably affect the outcome of a measurement.

As has been shown elsewhere, temperature gradients can lead to sample convection [1-3]. This has a strong effect on $B_{0}$-field gradient selected experiments such as for the measurement of diffusion coefficients $[1,4]$ and NOE enhancements [5]. We will not consider these effects further here as they have been reported widely elsewhere.

\section{Measurement of the sample temperature}

Temperature measurements were made using thullium (III) 1,4,7,10-tetraazacyclododecane-1,4,7,10-tetrakis(methylene phosphonate), which is referred to by the abbreviation, TmDOTP ${ }^{5-}$ [6-8] and has the molecular structure shown in Fig. 1. The sample used for all NMR measurements consisted of $0.2 \mathrm{M} \mathrm{TmDOTP}^{5-}$ in deuterated water; the ${ }^{1} \mathrm{H}$ spectrum of this sample is 


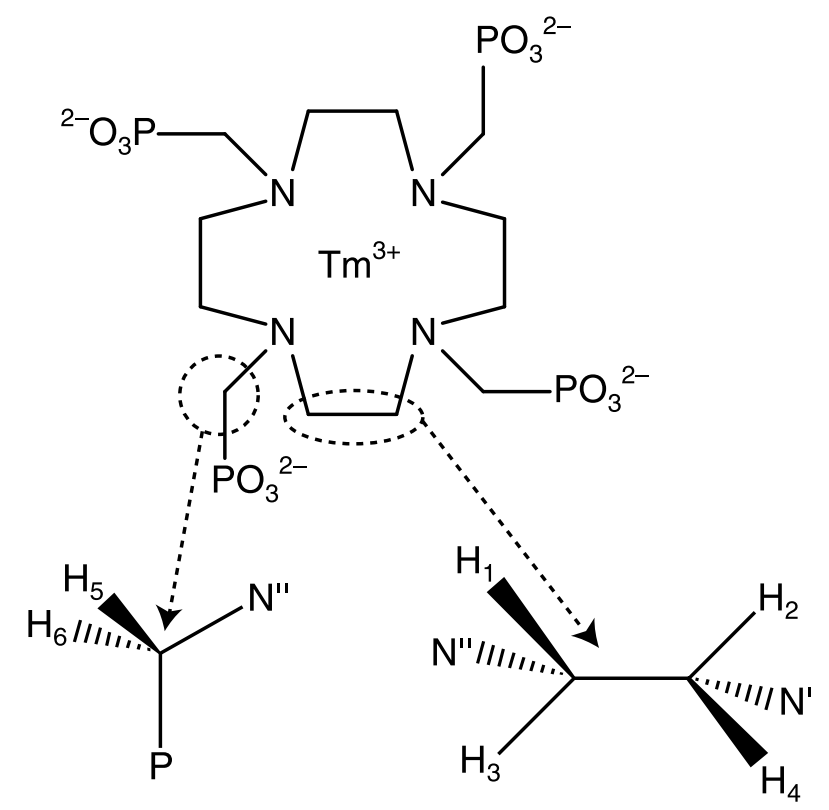

Fig. 1. The molecular structure of $\operatorname{TmDOTP}^{5-}$, showing the numbering system used. For details concerning the molecular conformation, see Ref. [6].

shown in Fig. 2. Due to symmetry, only six resonances are observed in the ${ }^{1} \mathrm{H}$ spectrum; the labelling scheme, shown in Fig. 1, and the assignment shown in Fig. 2 are taken from [6]. The thullium atom is paramagnetic and this leads to both very fast relaxation $\left(T_{1}<2 \mathrm{~ms}\right)$ and the large proton shifts seen in the spectrum.

The paramagnetic shifts of the protons in TmDOTP $^{5-}$ depend very strongly on temperature $[7,8]$. In this paper, the frequency separation between the peaks corresponding to $\mathrm{H}_{3}$ and $\mathrm{H}_{6}$ was used for determining the temperature. Of the six $\operatorname{TmDOTP}^{5-}{ }^{1} \mathrm{H}$ resonances, these two peaks were used because they have the narrowest lines, are always within the achievable spectral width for the temperatures used in our experiments, and because their chemical shifts move in opposite directions as the temperature increases. Using the separation between two peaks rather than the chemical shift of a single peak eliminates the need to reference the spectrum and, in imaging experiments, removes the influence of magnetic field inhomogeneities.

The actual temperature, $T$, of the sample when in the spectrometer was measured by introducing a thermocouple directly into the active region of the solution within the sample tube. After removing the thermocouple, the TmDOTP ${ }^{5-}{ }^{1} \mathrm{H}$ spectrum was measured under identical conditions. In this way, the frequency separation between $\mathrm{H}_{3}$ and $\mathrm{H}_{6}(\delta$, in ppm) was measured at several temperatures. In theory, $\delta$ should have a $T^{-1}$ dependence due to paramagnetic shielding as well as $T^{-1}$ and $T^{-2}$ dependencies due to pseudocontact interactions [8]. In practice, the equation:

$$
\delta=C \frac{1}{T}+D
$$

adequately described our data; adding a $T^{-2}$ term did not result in a better model. The best-fit values of the constants $C$ and $D$ for our $0.2 \mathrm{M} \mathrm{TmDOTP}^{5-}$ in deuterated water sample were $1.322( \pm 0.001) \times 10^{-5} \mathrm{~K}$ ppm and $-218.9( \pm 0.2) \mathrm{ppm}$, respectively. Since the spectrum of TmDOTP ${ }^{5-}$ depends on $\mathrm{pH}$ as well as temperature [8], this calibration will depend on concentration to some extent.

Although measurements of the absolute temperature are limited by the accuracy with which the constants $C$ and $D$ are specified, measurements of the deviation of the temperature are limited by the precision with which $\delta$ can be measured. It is possible to estimate this based on the signal-to-noise ratio of the peaks, their linewidths, the ratio of the peak heights, the frequency separation of the peaks, and the digitization of the spectrum [9]. For our data, such an analysis indicates that, despite the broadness of the peaks, the precision with which the frequency separation can be measured should be better than $0.5 \mathrm{~Hz}$. Consequently, differences in temperature on the order of $1 \mathrm{mK}$ should be detectable even though the absolute temperature is only calibrated to $\pm 0.1 \mathrm{~K}$.

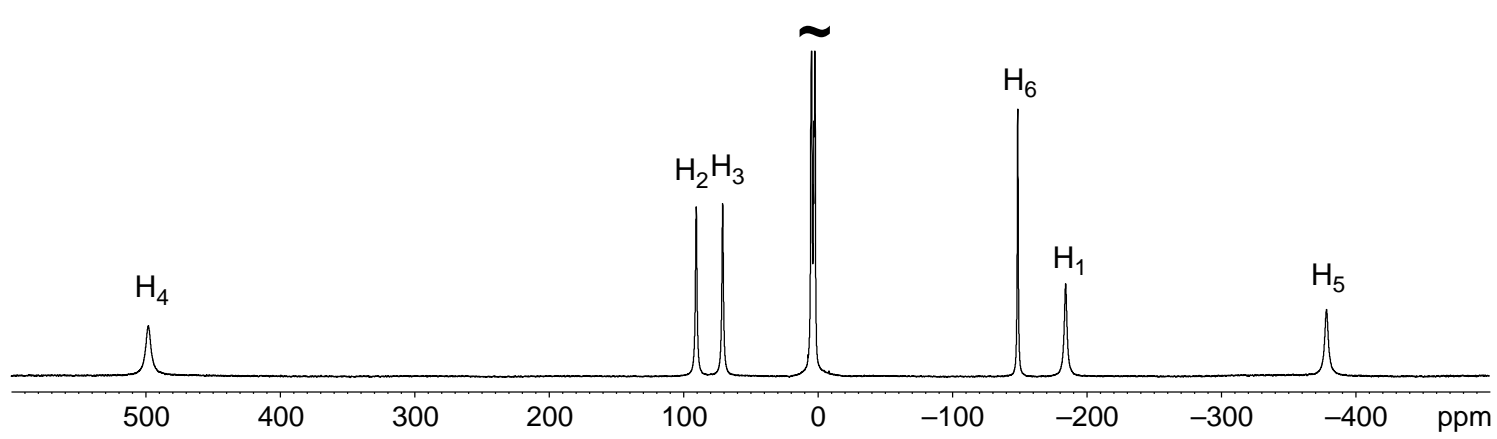

Fig. 2. $600 \mathrm{MHz}{ }^{1} \mathrm{H}$ spectrum of TmDOTP ${ }^{5-}$ in $\mathrm{D}_{2} \mathrm{O}$. The large peak at $\sim 5 \mathrm{ppm}$ is due to HOD and other impurities. 


\section{Systematic temperature deviations}

Although the variable-temperature (VT) control systems of many modern solution-state NMR spectrometers are capable of reporting and maintaining the temperature with a precision of $\pm 0.1 \mathrm{~K}$, this precision often underestimates the deviation between the system temperature (i.e. that reported by the spectrometer) and the sample temperature. The extent to which this deviation results in inaccuracies in physical measurements depends on both the magnitude of the deviation and the temperature dependence of the parameter being measured. For example, near room temperature the diffusion coefficient of camphene in deuteromethanol changes by $1 \%$ for every change in temperature of $0.5 \mathrm{~K}$.

In most solution-state NMR spectrometers, the sample temperature is maintained by flowing a heating or cooling gas over the sample tube. Usually, as was the case for the spectrometers used in this study, this gas (the VT gas) is chilled to below ambient temperature and then heated as it flows through the probe toward the sample. Typically, a thermocouple (or thermistor) is located just below the sample tube and in the flow of the VT gas. A feedback loop is used to regulate the power input to the heater so that the temperature, as registered by the thermocouple, is that set by the operator. Due to the finite heat capacity of the VT gas, the physical separation between the thermocouple and the active region of the sample results in a systematic deviation between the system temperature and the sample temperature. The size of this deviation depends on the design of the probe, how far the system temperature is from ambient, the VT gas flow rate, and the heating of the sample due to radiofrequency irradiation.

In the design of the NMR probe, the size of the separation between the thermocouple and the sample affects the temperature deviation; larger separations can be expected to result in larger deviations. The pathway used to flow the VT gas past the thermocouple and the sample is another factor that will influence both the deviation and the temperature gradients. Due to space constraints in narrow-bore magnets, most modern highresolution solution-state NMR probes have a relatively simple pathway for the VT gas so that there is enough room in the probe for the gradient coils, radiofrequency coils, and sample. Usually, the gas flows linearly from the bottom of the probe to the top, flowing first past the thermocouple, then the sample, and then finally out of the top of the probe. For example, in the Bruker probes used in this study the VT gas flows through a transfer line from the bottom of the probe. Before it reaches the sample, the VT gas is split between three different pathways that separately heat/cool the two radiofrequency coils and the sample; these pathways are separated by concentric glass cylinders that help support the radiofrequency coils. The pathways recombine near the top of the probe and the VT gas then flows out of the top of the probe.

Using $\mathrm{TmDOTP}^{5-}$ as an internal thermometer reveals that, at temperatures above ambient, the system temperature is lower than the sample temperature; the data are shown in Fig. 3. This effect is more pronounced for slower flow rates than for higher flow rates. At the highest temperature and the lowest flow rate, the sample (a)

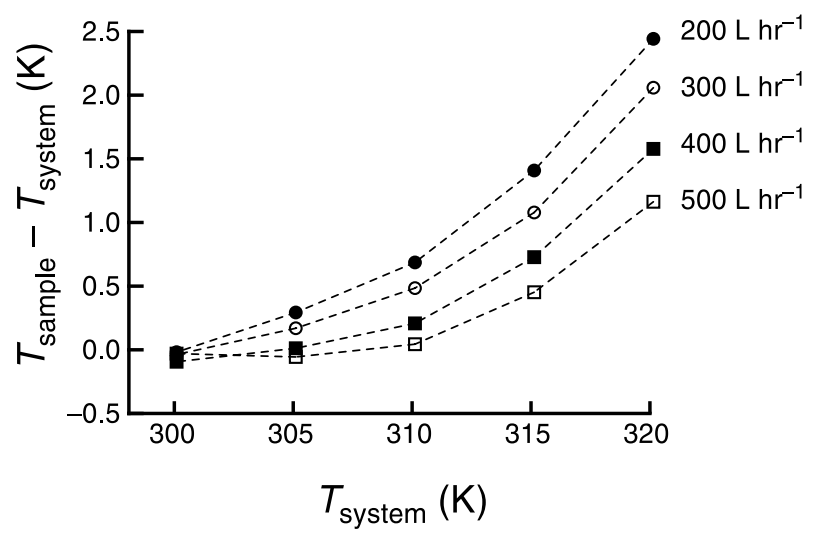

(b)

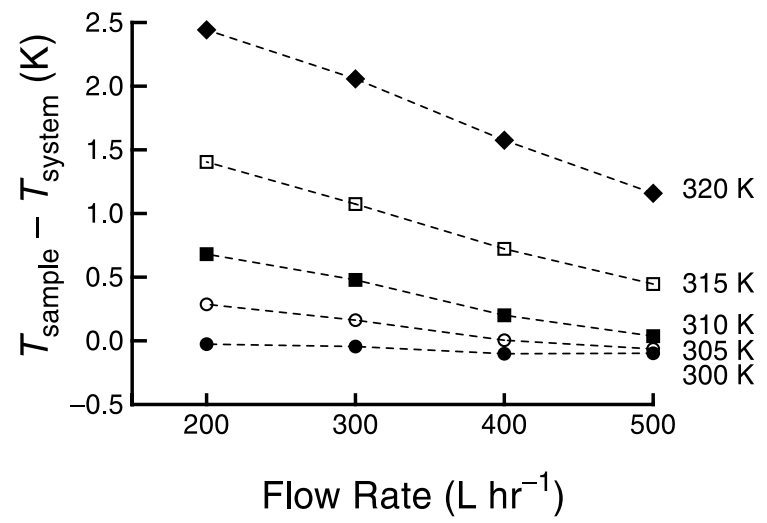

Fig. 3. The deviation between the system temperature and the sample temperature as (a) a function of the system temperature for several different VT gas flow rates and (b) a function of the flow rate for several system temperatures. In the first graph, gas flow rates of $200,300,400$, and $500 \mathrm{~L} \mathrm{~h}{ }^{-1}$ are indicated by dots, open dots, squares, and open squares, respectively. In the second graph, system temperatures of $300,305,310$, 315 , and $320 \mathrm{~K}$ are represented by dots, open dots, squares, open squares, and diamonds, respectively. The dotted lines are only a visual guide and do not imply a fit to the data. The data were acquired on a Bruker Avance spectrometer operating at $500 \mathrm{MHz}$ for ${ }^{1} \mathrm{H}$ and equipped with a 5 -mm $z$-axis gradient triple resonance inverse probe. The temperature regulation system was able to monitor and control the system temperature with a precision of $0.1 \mathrm{~K}$. 


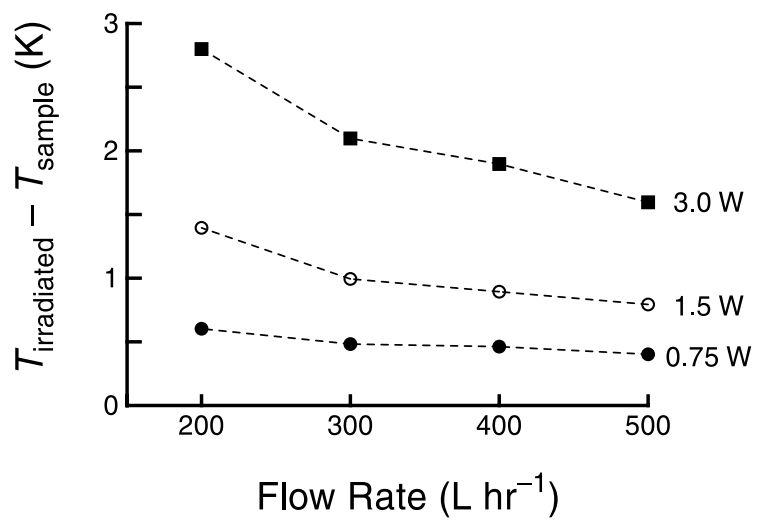

Fig. 4. The increase in the sample temperature due to radiofrequency irradiation at three field strengths. The sample temperature after $30 \mathrm{~s}$ of ${ }^{13} \mathrm{C}$ radiofrequency irradiation at $0.75,1.5$, and $3 \mathrm{~W}$ are indicated by dots, open dots, and squares, respectively. These powers correspond to radiofrequency field strengths of $1.1,1.5$, and $2.2 \mathrm{kHz}$, respectively, and the results shown are the means of measurements at five temperatures $(300,305,310,315$, and $320 \mathrm{~K})$. The dotted lines are only a visual guide and do not imply a fit to the data. Other than the period of radiofrequency irradiation prior to and during signal acquisition, the data were acquired under the same conditions as the data shown in Fig. 3.

temperature is almost $2.5 \mathrm{~K}$ higher than the system temperature.

Decoupling, particularly broadband decoupling of low $\gamma$ nuclei, requires high-power radiofrequency irradiation; this irradiation can be a significant source of sample heating [10]. We conducted a series of experiments in which the WALTZ-16 composite-pulse decoupling sequence was applied on the ${ }^{13} \mathrm{C}$ channel for $30 \mathrm{~s}$ prior to and during the acquisition of the
TmDOTP $^{5-}$ spectrum. The experiment was repeated three times with decoupling power levels of $0.75,1.5$, and $3 \mathrm{~W}$ (correspond to ${ }^{13} \mathrm{C}$ radiofrequency field strengths of $1.1,1.5$, and $2.2 \mathrm{kHz}$ ). We found that the increase in the sample temperature due to radiofrequency heating was not significantly affected by the system temperature. However, as shown in Fig. 4, lower heating gas flow rates resulted in larger increases in temperature than higher flow rates. These results indicate that higher flow rates are better at maintaining the sample temperature. Note that the increases shown in Fig. 4 are the differences between the sample temperature before and after radiofrequency irradiation; any deviations between the sample temperature and the system temperature will be additional to this.

\section{Temperature gradients}

The presence of temperature gradients in the sample are a common occurrence in NMR spectrometers. However, in most cases temperature gradients due not lead directly to noticeable inaccuracies in the measurement of physical parameters. This is because the measured value of a parameter that varies linearly with temperature is characteristic of the average temperature of the sample. Although most physical parameters have a non-linear dependence on temperature, it is usually safe to make the approximation that the temperature dependence is linear over the relatively small ranges of temperatures that arise in NMR samples under normal conditions (less than $1 \mathrm{~K} \mathrm{~cm}^{-1}$ ) and, therefore, the

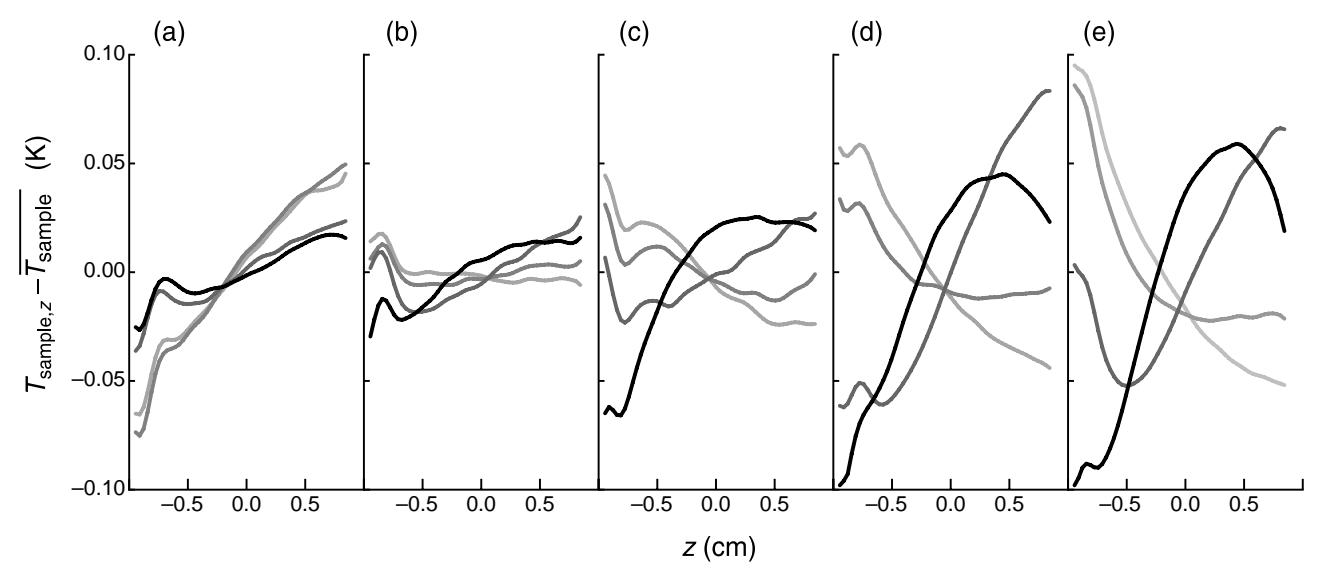

Fig. 5. The sample temperature profile as a function of the system temperature and the VT gas flow rate. The profiles show the deviation of the sample temperature from the mean sample temperature as a function of position along the $z$-axis. From left to right, the graphs show data for system temperatures of (a) $300 \mathrm{~K}$, (b) $305 \mathrm{~K}$, (c) $310 \mathrm{~K}$, (d) $315 \mathrm{~K}$, and (e) $320 \mathrm{~K}$. For each temperature, several profiles were acquired corresponding to VT gas flow rates of 200,300,400, and $500 \mathrm{~L} \mathrm{~h}^{-1}$. Darker lines represent lower flow rates, and $-z$ corresponds to the lower end of the sample tube. The data were acquired on the same instrument as described in Fig. 3 using a chemical shift imaging sequence. Despite collecting 4 transients for each of the 64 increments in the indirect (imaging) dimension, the total experiment time was only $22 \mathrm{~s}$ due to the short longitudinal relaxation time for the TmDOTP ${ }^{5-1} \mathrm{H}$ resonances. 
presence of a temperature gradient will not appreciably affect the outcome of a measurement.

For example, the apparent diffusion coefficient for a sample of camphene in deuteromethanol with an average temperature of $300 \mathrm{~K}$ and a temperature gradient of $1 \mathrm{~K}$ across the sample will differ by less than $0.01 \%$ from the diffusion coefficient measured for a sample with the same average temperature but no temperature gradient. In fact, a temperature gradient of $25 \mathrm{~K} \mathrm{~cm}^{-1}$ is necessary before the apparent diffusion coefficient deviates by more than $1 \%$ from its true value. Nevertheless, it is still important to minimize temperature gradients in the sample because, as mentioned in Section 1, temperature gradients can lead to convection which has a very large impact on certain types of experiments.

A chemical shift imaging experiment was used to detect sample temperature gradients. After Fourier transforming both dimensions, the peak positions in the direct (chemical shift) dimension were used to estimate the sample temperature as a function of position along the imaging axis. The resulting temperature profiles for several VT gas flow rates at system temperatures between 300 and $320 \mathrm{~K}$ are shown in Fig. 5.

The range of temperatures present in the profiles as a function of the system temperature for several VT gas flow rates are shown in Fig. 6; it is apparent that as the temperature increases the temperature range increases. Also, intermediate VT gas flow rates are better at maintaining the sample at a uniform temperature than the more extreme flow rates of 200 and $500 \mathrm{~L} \mathrm{~h}^{-1}$. The data at $300 \mathrm{~K}$ do not follow the same pattern as the rest of the data because, due to other sources of heat inside the magnet the "ambient" temperature inside the probe is $\sim 305 \mathrm{~K}$.

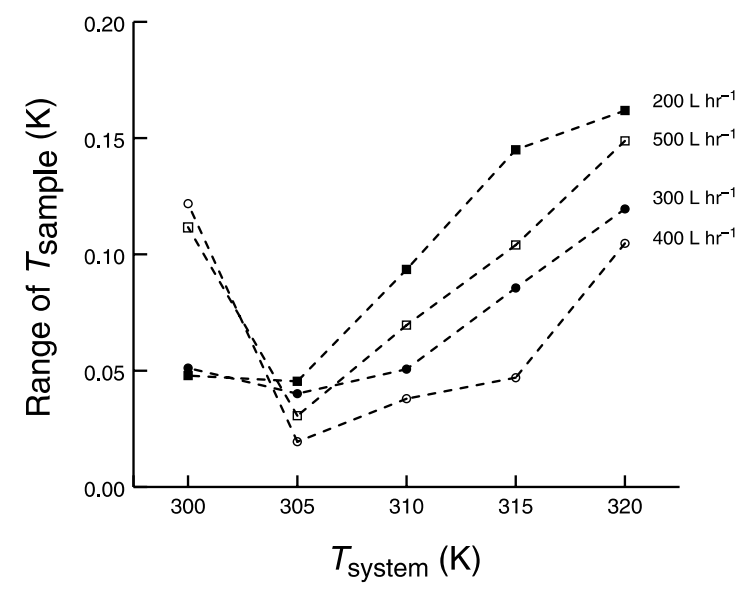

Fig. 6. The dependence of the range of temperatures in the sample on the system temperature and the VT gas flow rate. Squares, open squares, dots, and open dots correspond to flow rates of 200, 300, 400, and $500 \mathrm{~L} \mathrm{~h}^{-1}$, respectively. The dotted lines only connect the data points; they do not imply a fit to the data. The data are derived from the profiles shown in Fig. 5.

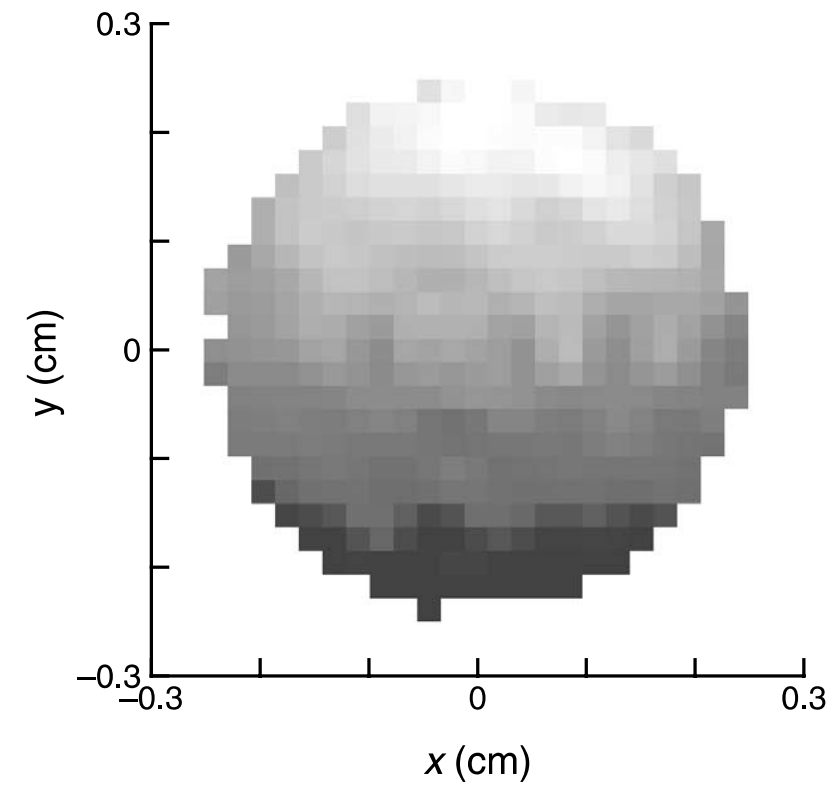

Fig. 7. The transverse temperature profile of a sample. Darker shades correspond to lower temperatures; the temperatures range from +0.14 to $-0.14 \mathrm{~K}$ from the average. The system temperature was $320 \mathrm{~K}$ and the VT gas flow rate was $400 \mathrm{~L} \mathrm{~h}^{-1}$. The profile was acquired using a Bruker Avance spectrometer operating at $600 \mathrm{MHz}$ for ${ }^{1} \mathrm{H}$ and equipped with a 5 -mm triple orthogonal gradient triple resonance inverse probe. The temperature regulation systems was able to monitor and control the system temperature with a precision of $0.1 \mathrm{~K}$.

In the temperature profiles shown in Fig. 5, the temperatures represent the average temperature in the $x y$-plane. To determine whether there were significant

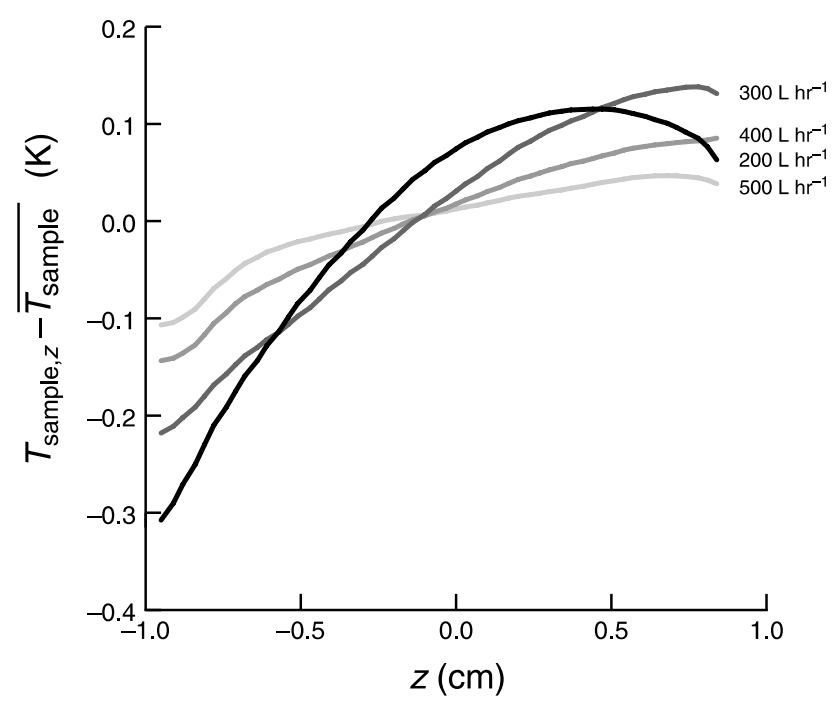

Fig. 8. The temperature profile due to low power $(1.1 \mathrm{kHz})$ WALTZ-16 decoupling. The decoupling was switched on for $30 \mathrm{~s}$ prior to imaging the sample and remained on throughout the experiment $(22 \mathrm{~s})$. The sample temperature was $320 \mathrm{~K}$ and the VT gas flow rate was varied between 200 and $500 \mathrm{~L} \mathrm{~h}^{-1}$; darker lines correspond to lower flow rates. The data were collected under the same conditions as for the data shown in Fig. 4. 

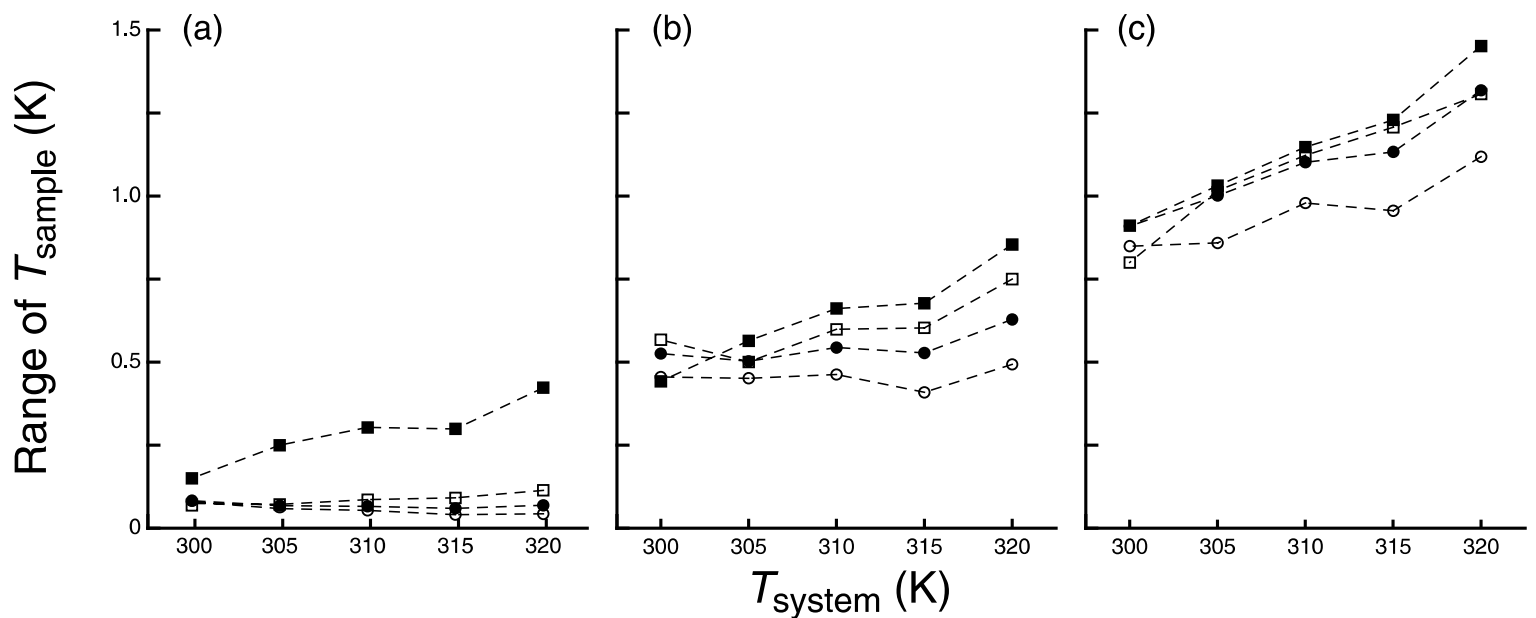

Fig. 9. The dependence of the range of temperatures in the sample on radiofrequency irradiation. (a), (b), and (c) correspond to ${ }^{13} \mathrm{C}$ radiofrequency irradiation at $0.75,1.5$, and $3.0 \mathrm{~W}$, respectively. The decoupling was switched on for $30 \mathrm{~s}$ prior to imaging the sample and remained on throughout the experiment (22 s). Squares, open squares, dots, and open dots correspond to VT gas flow rates of 200, 300, 400, and $500 \mathrm{~L} \mathrm{~h}^{-1}$, respectively. The dotted lines only connect the data points; they do not imply a fit to the data. The data were collected under the same conditions as those shown in Fig. 8.

temperature variations in the transverse plane, a threedimensional chemical-shift imaging experiment was performed. The resulting two-dimensional transverse temperature profile is shown in Fig. 7. This profile shows that the temperature varies much more along the $y$-axis than along the $x$-axis. We have also generated three-dimensional temperature profiles using a four-dimensional chemical shift imaging experiment; these profiles do not reveal any features that are not already indicated in the one- and two-dimensional profiles.

As the radiofrequency coil only covers part of the sample, the heating of the sample due to radiofrequency irradiation is uneven and results in a large temperature gradient. The temperature profile for three different decoupling power levels is shown in Fig. 8; these profiles have much larger temperature ranges than those shown in Fig. 5. The shape of these profiles is, for the most part, the same regardless of the system temperature and the VT gas flow rate. These temperature profiles are consistent with the expectation that the VT gas acts to cool the sample and that the direction of the gas flow is from the bottom of the tube to the top. As can be seen in Fig. 9, the range of temperatures in the sample due to radiofrequency irradiation depends weakly on the VT gas flow rate and the sample temperature. In all cases, higher flow rates are better at maintaining the sample at a uniform temperature than lower flow rates.

\section{Conclusion}

The actual temperature of the sample in solutionstate NMR spectrometers can deviate from the temperature reported by the spectrometer by several degrees. Whether this is actually a matter of concern depends on the desired measurement accuracy as well as the sensitivity of the physical constant to temperature. Although the exact size of the deviation between the sample temperature and the system temperature depends on the design of the probe and the temperature control system, in general, running experiments at temperatures other than ambient, low VT gas flow rates, and high-power radiofrequency decoupling can all be expected increase the discrepancy between the sample temperature and the system temperature.

The magnitude of the temperature gradients in the sample depends on the same parameters as those that cause a deviation between the sample temperature and the system temperature. Fortunately, as long as the average temperature of the sample is known, under most circumstances these gradients will not lead directly to noticeable errors in the measurements of physical constants. Nevertheless, for certain experiments, sample convection due to a temperature gradient in the sample can cause large measurement errors and, therefore, it is still useful to find experimental conditions under which the temperature gradients are minimized.

\section{Acknowledgments}

We thank Chun-wa Chung (GlaxoSmithKline, Stevenage, UK) for providing access to a spectrometer equipped with triple-orthogonal gradients, David Neuhaus (MRC Laboratory of Molecular Biology, Cambridge, UK) for generously providing instrument time to replicate our measurements on a second instrument (a Bruker DRX300), A. Dean Sherry (Southwestern Medical Center, University of Texas, USA) for a generous gift of a sample of $\mathrm{TmDOTP}^{5-}$, and Anthony 
Bielecki for helpful comments. N.M.L. thanks the Winston Churchill Foundation (USA), the Overseas Research Studentship Scheme (UK), and the National Science Foundation (USA) for support. This material is based on work supported under a National Science Foundation Graduate Research Fellowship.

\section{References}

[1] W.J. Goux, L.A. Verkruyse, S.J. Salter, The impact of RayleighBenard convection on NMR pulsed-field-gradient diffusion measurements, J. Magn. Reson. 88 (1990) 609-614.

[2] N.M. Loening, J. Keeler, Measurement of convection and temperature profiles in liquid samples, J. Magn. Reson. 139 (1999) 334-341.

[3] A. Jerschow, Thermal convection currents in NMR: flow profiles and implications for coherence pathway selection, J. Magn. Reson. 145 (2000) 125-131.
[4] A. Jerschow, N. Müller, Suppression of convection artifacts in stimulated-echo diffusion experiments: double-stimulated-echo experiments, J. Magn. Reson. 125 (1997) 372-375.

[5] A. Jerschow, N. Müller, Convection compensation in gradient enhanced nuclear magnetic resonance spectroscopy, J. Magn. Reson. 132 (1998) 13-18.

[6] C.F.G.C. Geraldes, A.D. Sherry, G.E. Kiefer, The solution structure of $\operatorname{Ln}(\mathrm{DOTP})^{5-}$ complexes. A comparison of lanthanide-induced paramagnetic shifts with the MMX energy-minimized structure, J. Magn. Reson. 98 (1992) 290-304.

[7] C.S. Zuo, J.L. Bowers, K.R. Metz, T. Nosaka, A.D. Sherry, M.E. Clouse, TmDOTP ${ }^{5-}$ : a substance for NMR temperature measurements in vivo, Magn. Reson. Med. 36 (1996) 955-959.

[8] C.S. Zuo, K.R. Metz, Y. Sun, A.D. Sherry, NMR temperature measurements using a paramagnetic lanthanide complex, J. Magn. Reson. 133 (1998) 53-60.

[9] P.E. Jupp, K.D.M. Harris, A.E. Aliev, Precision in estimating the frequency separation between spectral lines, J. Magn. Reson. 135 (1998) 23-29.

[10] S.R. Maple, A. Allerhand, Ultra-high-resolution NMR. IV. A simple technique for measurements of sample temperature gradients, J. Magn. Reson. 66 (1986) 168-171. 